\title{
QUANTUM COMPUTING WITH PARTICLE IN A DRIVEN SQUARE WELL
}

\author{
Shahid Iqbal ${ }^{1,2}$ and Farhan Saif ${ }^{2}$ \\ ${ }^{1}$ Max-Planck Institute for the Physics of Complex Systems \\ Noethnitzer Strasse 38, Dresden 01187, Germany \\ 2 Department of Electronics \\ Quaid-i-Azam University \\ Islamabad 45320, Pakistan \\ e-mails: ishahid @ ele.qau.edu.pk saif @ fulbrightweb.org
}

\begin{abstract}
We show that a particle confined in a one-dimensional square well in the presence of an external periodic linear modulation undergoes coherent oscillations between the discrete levels analogous to an atom exposed to an electromagnetic field. We define the selection rules for state transitions based on the matrix elements of the perturbation potential. These selection rules contribute to the time evolution of the qubit which can partake efficiently in quantum informatics. Quantum logic gates are realized in the square-well potential by controlling the period of modulation.
\end{abstract}

Keywords: quantum computation, quantum well, qubit, artificial atom, coherent oscillation, quantum logic gate, transition matrix element, selection rules, quantum informatics, probability amplitude.

\section{Introduction}

A quantum computer performs computation and information processing based on quantum mechanics. The study of quantum computers, a vital branch of quantum information [1-3], first attracted the attention of researchers in the seventies and eighties, when the precision of experiments, especially in atomic physics and quantum optics, made the control of quantum systems achievable. Any two-level quantum system, in principle, can define a qubit; however, the availability of quantum-logic-gate operations, long decoherence times, and the presence of an efficient way to measure the output make a physical system suitable for building quantum computers. Among the first implementations, there were the nuclear spins of molecules using the nuclear-magnetic-resonance (NMR) techniques which enabled the implementations of Shor's algorithm with seven qubits [4] and simulation of quantum maps with three qubits [5]. Some other physical systems proposed as quantum computers include Bose-Einstein condensates (BEC) of cold atoms in optical lattices [6], ion traps [7] in atomic physics, Josephson junctions [8-12] and quantum dots $[13,14]$ in solid state physics, and multilevel systems, for instance, Rydberg's atoms [15].

With the availability of semiconductor technologies that grow layers epitaxially [16] with atomic-scale accuracy, material bandgaps can be designed [17] to confine the electrons in much the same way as described by a one-dimensional square well [18]. The confinement of electrons in such a quantum well

Manuscript submitted by the authors in English on July 31, 2008. 
is regarded as an artificial atom. There is good analogy in performing quantum computation through natural atoms and artificial atoms since both have discrete energy levels and both exhibit coherent quantum oscillations between the levels. In most natural atoms or molecules, the bound states have well-defined parities, and the electric-dipole selection rules forbid transitions between states with the same parity. In contrast to naturally occurring atoms, artificial atoms can be designed to attain specific characteristics. For example, if the symmetry of the potential is broken, the bound states of the artificial atom lose their well-defined parities. As a result, all the electric-dipole matrix elements could be nonzero and every transition could be possible [19-21].

In the present article, we discuss a one-dimensional square well in the presence of external periodic modulation as an appropriate physical system to implement quantum computers. We show that the modulation causes transitions between the eigenstates of the initial stationary quantum system and calculate the matrix elements corresponding to linear and quadratic modulations applied to the base of the square well. This leads us to define the selection rules for the state transitions. Based on these selection rules, we construct a two-level quantum system forming the desired qubit. The qubit in the periodically modulated square well is capable of performing computation and information processing.

This paper is organized as follows. In Sec. 2, we give a brief review of the one-dimensional square well. In Sec. 3, we discuss the effect of external modulation on the stationary states of unmodulated systems. The matrix elements and selection rules for the state transitions are discussed in Sec. 4. Based on these results, in Sec. 5 we discuss quantum informatics in a driven square well.

\section{One-Dimensional Box: A Pico Review}

The dynamics of a particle of mass $m$ confined in a one-dimensional infinite square well of length $2 a$ is governed by the Hamiltonian

$$
\hat{H}_{0}=\frac{\hat{p}^{2}}{2 m}+\hat{V}(x)
$$

where $\hat{V}(x)$ is the potential with reflection symmetry such that $\hat{V}(x)=0$ for $|x|<a$ and $\infty$ otherwise. Consequently, the Hamiltonian $\hat{H}_{0}$ is invariant under reflection and obeys $\left[\hat{H}_{0}, \hat{I}\right]=0$, where $\hat{I}$ denotes the parity operator such that $\hat{I} \hat{V}(x)=\hat{V}(-x)$. This means that eigenstates of $\hat{H}_{0}$, denoted by $|n\rangle$, can simultaneously be eigenstates of the parity operator $\hat{I}$, i.e., $\hat{I}|n\rangle=\lambda_{i}|n\rangle$, where the eigenvalue $\lambda_{i}= \pm 1$. Here, $\lambda_{i}=+1$ denotes eigenstates with even parity and $\lambda_{i}=-1$ indicates eigenstates with odd parity. The eigenstates $|n\rangle$ of $\hat{H}_{0}$ and $\hat{I}$ are

$$
\langle x \mid n\rangle \equiv\left\{\begin{array}{l}
\sqrt{\frac{1}{a}} \sin \left(\frac{n \pi x}{2 a}\right), \quad \text { when } \quad n=2,4,6, \ldots, \\
\sqrt{\frac{1}{a}} \cos \left(\frac{n \pi x}{2 a}\right), \quad \text { when } \quad n=1,3,5, \ldots
\end{array}\right.
$$

From Eq. (2), it is clear that for odd quantum number $n$ the eigenstates $|n\rangle$ have even parity, whereas for even quantum number $n$, the eigenstates $|n\rangle$ have odd parity. A superposition of the stationary eigenstates $|n\rangle$, that is, $\left|\psi_{0}\right\rangle=\sum_{n} a_{n}|n\rangle$, indicates an arbitrary wave packet which evolves in time as

$$
\left|\psi_{0}(t)\right\rangle=\sum_{n} a_{n}|n\rangle e^{-i E_{n} t / \hbar}
$$


where $a_{n}$ is the time-independent probability amplitude of the wave packet and $E_{n}=\frac{n^{2} \hbar^{2} \pi^{2}}{8 m a^{2}}$ denotes the eigenenergy corresponding to $\hat{H}_{0}$, such that $\hat{H}_{0}|n\rangle=E_{n}|n\rangle$. For simplicity in our analysis, we assume that the quantum-well walls are infinitely high; nevertheless, we may replace infinite walls by some sufficiently high finite potential $V_{0}$ in order to realize the quantum system physically.

\section{Driven One-Dimensional Box}

The dynamics of a quantum particle in a one-dimensional square well in the presence of a weak external periodic modulating force is governed by the Hamiltonian

$$
\hat{H}=\hat{H}_{0}+\lambda \hat{x}^{\alpha} \cos \omega t
$$

where $\alpha$ defines the spatial-dependence law of the external time-periodic modulation of amplitude $\lambda$ and frequency $\omega$. For $\alpha=1$, we have a square well with periodically-changing linear potential at the base of the square well, and $\alpha=2$ corresponds to the periodically-changing quadratic potential at the base of the square well. Moreover, $\hat{H}_{0}$ describes the dynamics of the undriven system, as defined in Eq. (1). The solution of the time-dependent Schrödinger equation

$$
i \hbar \frac{\partial}{\partial t}|\psi(t)\rangle=\left(\hat{H}_{0}+\lambda \hat{x}^{\alpha} \cos \omega t\right)|\psi(t)\rangle
$$

is

$$
|\psi(t)\rangle=\sum_{n} a_{n}(t)|n\rangle e^{-i E_{n} t / \hbar}
$$

where $a_{n}(t)$ are time-dependent probability amplitudes which evolve in time as

$$
i \hbar \frac{\partial}{\partial t} a_{m}(t)=\lambda \cos \omega t \sum_{n} a_{n}(t) V_{m n} e^{i \omega_{m n} t} .
$$

In this description, we used the fact that

$$
\sum_{n} a_{n}(t)|n\rangle \frac{\partial}{\partial t} e^{-i E_{n} t / \hbar}=\sum_{n} a_{n}(t) \hat{H}_{0}|n\rangle e^{-i E_{n} t / \hbar}
$$

revealed from the time-dependent Schrödinger equation corresponding to $\hat{H}_{0}$, along with the orthogonality condition $\langle m \mid n\rangle=\delta_{m n}$. Here, $\omega_{m n}=\left(E_{m}-E_{n}\right) / \hbar$ is the transition frequency for the transition from $E_{n}$ to $E_{m}$ and $V_{m n}=\left\langle m\left|\hat{x}^{\alpha}\right| n\right\rangle$ is the matrix element which controls the transition probability from the state $|n\rangle$ to the state $|m\rangle$; below we refer to $|n\rangle$ as a reference state and $|m\rangle$ as a target state.

For a small time-dependent modulation considered as a perturbation to the initial system described by the Hamiltonian $\hat{H}_{0}$, the time-dependent probability amplitude of the wave packet $a_{n}(t)$ can be approximated, in view of the time-dependent perturbation theory [18]. In the presence of a weak timeperiodic modulation, the secular perturbation theory [23] leads to the solution for $a_{n}(t)$. We study classical nonlinear resonances in quantum systems [24-28], where the $N$ th primary resonance occurs when the time required for one cycle of unmodulated motion is nearly equal to $N$ cycles of external modulation $[23,29]$. Since the energy is no longer a constant of motion, we calculate quasienergy eigenstates and quasienergy eigenvalues for the time-periodic system [30]. Following this analysis, we find quasienergy states as Floquet states and quasi-energies as characteristic values of the Matheiu differential equation [31]. 


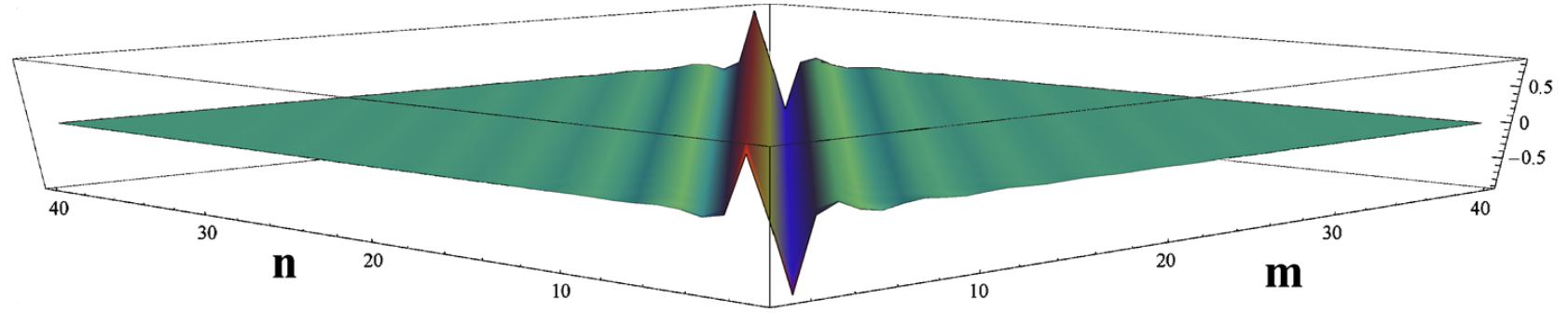

Fig. 1. Transition matrix elements of linear potential applied at the base of the square well. The diagonal matrix elements are zero and only the nearest neighboring off-diagonal elements contribute significantly to the transitions.

\section{Transition Selection Rules}

The external modulation induces the transitions between the stationary states of the square well. The transition between the state $|n\rangle$ and the state $|m\rangle$ can only be possible if the corresponding matrix element, defined as

$$
\left\langle n\left|\hat{x}^{\alpha}\right| m\right\rangle=\int_{-a}^{a} \varphi_{n}(x)^{*} \hat{x}^{\alpha} \varphi_{m}(x) d x,
$$

is nonzero. In view of the discussion in Sec. 2, the eigenstates of $\hat{H}_{0}$ have definite parity due to the reflection symmetry of the box potential $\hat{V}(x)$. The matrix elements depend on the overall parity of the reference state $|n\rangle$, the target state $|m\rangle$, and the operator associated with the perturbation that causes the transitions. The matrix elements given by Eq. (8) have nonzero values only if the collective parity of states $|n\rangle,|m\rangle$, and operator $\hat{x}^{\alpha}$ is even. Hence, there exist selection rules that forbid transitions between certain states, depending on the parity of the reference state $|n\rangle$, the target state $|m\rangle$, and the perturbation-potential index $\alpha$.

For a linear time-dependent potential, i.e., $\alpha=1$, Eqs. (2) and (8) lead to the conclusion that coupling between the same parity states (i.e., quantum number $n$ and $m$ both even or both odd) does not exist. Hence, the matrix elements are nonzero only for the opposite-parity states ( $n$ even and $m$ odd or $n$ odd and $m$ even), as one can see in Fig. 1. Using Eq. (2) and integrating Eq. (8), one obtains the matrix elements as follows:

$$
\langle n|\hat{x}| m\rangle= \begin{cases}0, & \text { when } m=n, \\ \frac{-4 a(-1)^{(m+n+1) / 2}}{\pi^{2}}\left(\frac{1}{(m-n)^{2}}+\frac{1}{(m+n)^{2}}\right), & \text { when } m \neq n .\end{cases}
$$

It is worth noting that the diagonal matrix elements are zero for a linearly-modulated square well due to the parity of the states. In view of Eq. (9), the selection rule for the linear potential states that $a$ transition from the state $|n\rangle$ to the state $|m\rangle$ takes place when $|n\rangle$ and $|m\rangle$ have opposite parity and it is forbidden when $|n\rangle$ and $|m\rangle$ have the same parity. It is further clear from Eq. (9) that for large values of the quantum number $n$, the nearest neighboring off-diagonal matrix elements are such that

$$
\langle n|\hat{x}| n+1\rangle \approx(-1)\langle n|\hat{x}| n-1\rangle .
$$

In contrast to the linear modulation, the parity of the quadratic perturbation is even, and one can conclude from Eqs. (2) and (8) that the matrix elements are zero for the states with opposite parity and 


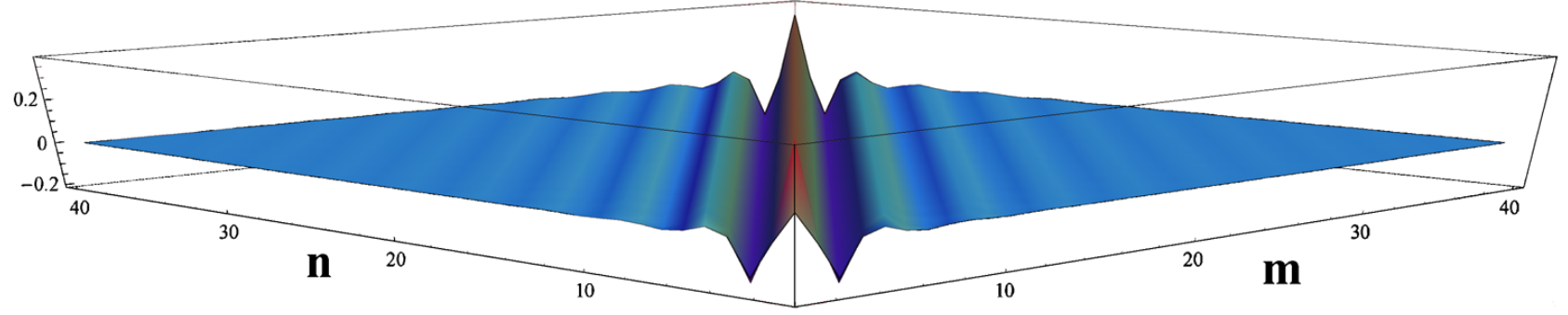

Fig. 2. Transition matrix elements of quadratic potential applied at the base of the square well. The diagonal matrix elements are nonzero in contrast to the case of linear potential. The off-diagonal matrix elements decrease rapidly with increase in the distance from the diagonal.

coupling exists only between the states with the same parity, as plotted in Fig. 2. For the states with the same parity, the matrix elements take the following values:

$$
\left\langle n\left|\hat{x}^{2}\right| m\right\rangle= \begin{cases}\frac{a^{2}}{3}+\frac{2 a^{2}(-1)^{n}}{n^{2} \pi^{2}}, & \text { when } m=n . \\ \frac{8 a^{2}(-1)^{(m-n) / 2}}{\pi^{2}}\left(\frac{1}{(m-n)^{2}}-\frac{1}{(m+n)^{2}}\right), & \text { when } m \neq n .\end{cases}
$$

Hence, for quadratic modulation the selection rule states that a transition from the state $|n\rangle$ to the state $|m\rangle$ takes place when $|n\rangle$ and $|m\rangle$ have the same parity, and it is forbidden when $|n\rangle$ and $|m\rangle$ have opposite parity. It is interesting that the diagonal elements take nonzero values and the nearest neighboring off-diagonal elements are approximately the same, i.e.,

$$
\left\langle n\left|\hat{x}^{2}\right| n+2\right\rangle \approx\left\langle n\left|\hat{x}^{2}\right| n-2\right\rangle,
$$

in contrast to the case of linear modulation.

\section{Quantum Informatics in a Driven One-Dimensional Box}

Particles confined in quantum wells possess discrete energy levels and may exhibit Rabi-like oscillations in the presence of external periodic modulations analogous to atomic or molecular systems [19]. Therefore, quantum wells can be considered as artificial atoms. In our discussion, we assumed a square well infinitely deep with tightly binding energy spectrum [32], where the level spacing increases linearly with increase in the principle quantum number $n$. For linear modulation at frequency $\omega$, the resonance oscillations with transition frequency $\omega_{0}=\left(E_{2}-E_{1}\right) / \hbar$ occur predominantly only between the states $|1\rangle$ and $|2\rangle$. The other levels of the system remain off-resonant and hence decoupled for this particular frequency due to increasing level spacing of the energy spectrum. Consequently, we get effectively a two-level quantum system that defines a qubit. The time evolution of the wave function for our two-level system is given by the expression

$$
\left|\psi_{0}(t)\right\rangle=e^{-i \varphi t}\left(a_{1}|1\rangle e^{i \omega_{0} t / 2}+a_{2}|2\rangle e^{-i \omega_{0} t / 2}\right),
$$

which is obtained from Eq. (3) by terminating the summation at $n=2$. Here, $\varphi=\left(E_{2}+E_{1}\right) / 2 \hbar$ and $a_{1}$ and $a_{2}$ are probability amplitudes such that $\left|a_{1}\right|^{2}+\left|a_{2}\right|^{2}=1$. 
The two-component vector $\left|\psi_{0}\right\rangle=\left[\begin{array}{l}a_{1} \\ a_{2}\end{array}\right]$ of this two-level system represents a qubit. When an external time-periodic field is applied, the probability amplitudes change with time, and one can perform single qubit operations by selecting particular interaction times.

In order to perform single-qubit-logic operations, one needs to know the time evolution of the probability amplitudes of the two-level system. As Eq. (7) shows, the time evolution of the probability amplitudes, in the present scenario, depends on the matrix elements of the applied external potential. The matrix elements for linear external modulation are expressed by Eq. (9). For our two-level system, the diagonal matrix elements are zero, i.e., $V_{i i}=V_{j j}=0$, and off-diagonal elements are $V_{j i} \approx V_{i j}=V$, where $V_{i j}=\langle i|x| j\rangle$ with $i, j=1,2$. In response to the external periodic modulation, the probability amplitudes evolve in time as

$$
\dot{a}_{1}(t)=\frac{\Omega}{2 i} a_{2}(t) e^{i \delta t}
$$

and

$$
\dot{a}_{2}(t)=\frac{\Omega}{2 i} a_{1}(t) e^{-i \delta t},
$$

where $\delta=\omega-\omega_{0}$ is the detuning of the two frequencies and $\Omega=\lambda V / \hbar$. Furthermore, the amplitude of the transition matrix elements $V \approx-4 a / \pi^{2}$. For $\delta=0$, the solutions of these coupled differential equations give the state of the system as follows:

$$
|\psi(t)\rangle=e^{-i \varphi t}\left(\cos \frac{\Omega t}{2} e^{i \omega_{0} t / 2}|1\rangle-i \sin \frac{\Omega t}{2} e^{-i \omega_{0} t / 2}|2\rangle\right) .
$$

We initially prepare our quantum system in the state $|1\rangle$, i.e, $\left|a_{1}(t=0)\right|^{2}=1$ and $\left|a_{2}(t=0)\right|^{2}=0$, then expose it to a linear external periodic modulation at frequency $\omega$ resonant with the transition frequency $\omega_{0}$, i.e, $\omega=\omega_{0}$. This external modulation coherently redistributes the probability amplitudes of our two-level system. The interaction time $\tau$ corresponding to the Hadamard gate is determined with the help of Eq. (14). Thus, for $\tau=\pi / \Omega$, expression (14) transforms to

$$
|\psi(\tau)\rangle=e^{-i \theta \tau}\left(\frac{|1\rangle-i e^{-i \omega_{0} \tau}|2\rangle}{\sqrt{2}}\right),
$$

which is the Hadamard gate, with $\theta=E_{1} / \hbar$. Similarly, using Eq. (14), we can perform the NOT operation i.e., $\left|a_{1}(\tilde{\tau})\right|^{2}=0$ and $\left|a_{2}(\tilde{\tau})\right|^{2}=1$, where $\tilde{\tau}$ is the interaction time for the NOT operation, which comes out to be $\tilde{\tau}=\pi / 2 \Omega$. In this way, we obtain quantum control of single-qubit operations by choosing an appropriate interaction time of the external modulation.

In the case of quadratic modulation, transitions are possible only between states with the same parity, as discussed in Sec. 4. Our two-level system comprises the states $|1\rangle$ and $|3\rangle$ with the transition frequency $\omega_{0}=\left(E_{3}-E_{1}\right) / \hbar$. It is explicitly clear from Eq. (10) that, for the two-level system composed of the states $|1\rangle$ and $|3\rangle$, the diagonal elements are nonzero. The optical selection rules [21] and the coherent population transfer between selected quantum states [22] of the analogous systems, in which all the matrix elements are nonzero, have recently been analyzed.

\section{Discussions}

We have presented artificial atoms, subject to linear and quadratic time-periodic modulations, to implement a quantum computer. The energy spectrum of the artificial atom is such that the transition 
frequency increases in magnitude with increase in the principle quantum number $n$, in contrast to the natural atoms where it decreases with increase in the quantum number $n$. The transitions can be tuned to the desired frequencies by changing the mirror symmetry of the potential defining the artificial atom. Furthermore, the qubits defined by these artificial atoms are scalable, which is the key requirement of large quantum circuits for performing more complex calculations. In quantum computation, logic operations are accomplished in a time much less than it takes for the interactions of the qubit with the environment. This is because interactions with the environment destroy the coherence of the state and convert it into a mixed state. The coupling of artificial atoms with the environment is relatively larger than the natural atomic systems; however, the tunability of transition frequencies and the scalability of qubits make the system more advantageous for performing quantum informatics.

\section{Acknowledgments}

The authors thank R. ul Islam, K. Naseer, M. Ayub, T. Abbas, M. Ali, and I. Rehman for many discussions and the Higher Education Commission of Pakistan for funding under Grants Nos. 041203721E026 and 20-230/RND/03143.

\section{References}

1. M. A Nielsen and I. L. Chuang, Quantum Computation and Quantum Information, Cambridge University Press (2000).

2. D. Bouwmeester, A. Ekert, and A. Zeilinger, The Physics of Quantum Information, Springer-Verlag, Berlin (2001).

3. G. Leuchs and T. Beth, Quantum Information Processing, Wiley, Weinheim (2003).

4. L. Vandersypen, M. Steffen, G. Breyta, et al., Nature, 414, 883 (2001).

5. Y. S. Weinstein, S. Lloyd, J. Emerson, and D. G. Cory, Phys. Rev. Lett., 89, 157902 (2002).

6. M. Greiner, O. Mandel, T. Esslinger, et al., Nature, 415, 39 (2002).

7. J. I. Cirac and P. Zoller, Phys. Rev. Lett., 74, 4091 (1995).

8. D. Vion, A. Aassime, A. Cottet, et al., Science, 296, 886 (2002).

9. Yu. Makhlin, G. Schon, and A. Shnirman, Rev. Mod. Phys., 73, 357 (2001).

10. Y. Nakamura, Yu. A. Pashkin, and J. S. Tsai, Nature, 398, 786 (1999).

11. Y. Yu, S. Han, X. Chu, et al., Science, 296, 889 (2002).

12. T. Yamamoto, Yu. A. Pashkin, O. Astafiev, et al., Nature, 425, 941 (2003).

13. T. Hayashi, T. Fujisawa, H. D. Cheong, et al., Phys. Rev. Lett., 91, 226804 (2003).

14. R. Hanson, B. Witkamp, L. M. K. Vandersypen, et al., Phys. Rev. Lett., 91, 196802 (2003).

15. J. Ahn, T. C. Weinacht, and P. H. Bucksbaum, Science, 287, 463 (2000).

16. A. Y. Cho, Appl. Phys. Lett., 19, 467 (1971).

17. J. P. van der Ziel, R. Dingle, R. C. Miller, et al., Appl. Phys. Lett., 26, 463 (1975).

18. R. W. Robinett, Quantum Mechanics: Classical Results, Modern Systems and Visaulized Examples, Oxford University Press, New York (2006).

19. J. Q. You and F. Nori, Phys. Today, 58, 42 (2005).

20. J. Clarke, Science, 239, 992 (1988).

21. Yu-xi Liu, J. Q. You, L. F. Wei, et al., Phys. Rev. Lett., 95, 087001 (2005). 
22. L. F. Wei, J. R. Johansson, L. X. Cen, et al., Phys. Rev. Lett., 100, 113601 (2008).

23. A. J. Lichtenberg and M. A. Lieberman, Regular and Stochastic Motion, Springer-Verlag, Berlin (1983).

24. M. E. Flatté and M. Holthaus, Ann. Phys. (N.Y.), 245, 113 (1996).

25. G. P. Berman and G. M. Zaslavsky, Phys. Lett. A, 61, 295 (1977).

26. F. Saif and M. Fortunato, Phys. Rev. A, 65, 013401 (2001).

27. F. Saif, Phys. Rep., 419, 207 (2005).

28. F. Saif, Phys. Rep., 425, 369 (2006).

29. L. E. Reichl, The Transition to Chaos: Conservative Classical Systems and Quantum Manifestations, Springer-Verlag, New York (2004).

30. H. Sambe, Phys. Rev. A, 7, 2203 (1973).

31. M. Abramowitz and I. A. Stegun, Handbook of Mathematical Functions, Dover, New York (1992).

32. S. Iqbal, Qurat-ul-Ann, and F. Saif, Phys. Lett. A, 356, 231 (2006). 